\title{
Infrared Studies of Protostellar Binaries
}

\author{
Tom Greene \\ NASA's Ames Research Center, M.S. 245-6, Moffett Field CA 94035, \\ $U S A$
}

\begin{abstract}
Although much progress has been made in studying the multiplicity properties of pre-main-sequence $\mathrm{T}$ Tauri stars over the past decade, younger and more embedded protostellar systems are just now being surveyed for multiplicity. I examine several recent infrared imaging and spectroscopic studies which reveal the multiplicity properties of embedded protostars for the first time. The overall binary / multiplicity fraction of protostars is statistically indistinguishable from that of premain-sequence stars when measured over similar separation distances. Some individual components of protostellar binaries appear to be coeval from their spectral energy distributions or spectra. However, there are some systems which appear to consist of both a protostar and a pre-mainsequence star, and the coevality of these systems is very uncertain. There is much yet to be learned about the multiplicity properties of protostars, and future high resolution near-IR imaging surveys will likely make much headway.
\end{abstract}

\section{Introduction}

Most nearby solar-type stars are in binary (or multiple) systems (Abt \& Levy 1976), and binaries are at least as common among younger pre-main-sequence (PMS) stars. Numerous recent studies have shown that the binary fractions of PMS stars in nearby dark clouds are $\sim 2$ times as high as for main sequence stars in the solar neighborhood (e.g., Ghez et al. 1997; Duchêne 1999; Melo 2003), although the more distant and massive Orion Trapezium cluster has a binary fraction that is similar to that of the local field (Petr et al. 1998). Thus it is likely that most sun-like stars are born as binaries, and the binary fraction of stars may change over time (and may be a function of environment as well).

However, the dynamical evolution of multiple systems is neither understood or constrained very well from existing observations of PMS and MS stars. It is very difficult to determine the initial environmental conditions and binary fractions of nearby field stars when they formed, so it is very difficult to understand how $\sim 10^{6}$ yr old PMS stars and binaries evolve dynamically as they evolve towards the main sequence. Molecular clouds disappear and their stars disperse after only a few million years, a tiny fraction of the median age of the local field population. However, it may be instructive to examine whether and how stars and binaries evolve dynamically between the time they form as protostars to 
when they are PMS stars (and when their stellar groupings and parental clouds disperse).

There have been several recent (near-) infrared (IR) observational studies which investigate the multiplicity properties of such embedded protostars and compare them to those of PMS stars, sometimes even in the same cloud. Besides probing dynamical evolution issues, these studies also illuminate other interesting aspects of binarity in some of the youngest stellar systems. These properties include coevality and similarity of accretion properties between binary members as well as establishing causal links between binarity and other properties such as jets or outflows. We now examine some of these recent works, their results, and their implications.

\section{Disintegrating Systems and Herbig-Haro flows}

Reipurth (2000) examined high resolution imaging data of sources driving HerbigHaro $(\mathrm{HH})$ objects and found that 11 or 12 of the 14 systems were multiple, a very high fraction of $\sim 80 \%$. Approximately half of the multiples appeared binary, and the others were higher order. $\mathrm{HH}$ objects are generally very young, and their central stars are often surrounded by substantial amounts of circumstellar matter (Reipurth et al. 1993) which causes large extinctions. Reipurth (2000) postulated that close interactions between the components in each system trigger accretion events, causing the strong $\mathrm{HH}$ outflows observed. Furthermore, many triple (or higher multiple) systems are likely to decay in only about $10^{4} \mathrm{yr}$ after formation. This should quickly result in an increase of single and binary stars. Although this dynamical evolution appears to be qualitatively consistent with the results of multiplicity surveys of PMS stars, it will be difficult to compare detailed predictions of this scenario with current observations because most of the dynamical evolution occurs at such early times.

\section{A New Protostellar Multiplicity Survey}

A series of two IR imaging studies have recently surveyed the multiplicity properties of other very young embedded systems. Haisch et al. (2002) imaged 19 Class I and flat-spectrum protostars in the nearby $\rho$ Oph and Ser clouds. NearIR $(J H K L ; 1.25-3.4 \mu \mathrm{m})$ direct imaging data were acquired for all 19 sources and supplemented with mid-IR $(N$ band; $11 \mu \mathrm{m})$ images for 12 objects. The near-IR data were generally seeing limited ( $\lesssim 1^{\prime \prime}$ resolution) and the mid-IR data were diffraction-limited ( $\sim 0.5$ resolution). Haisch et al. (2003) have recently greatly expanded this survey to a total of 91 Class I and flat-spectrum protostars in nearby clouds. The new observations consist of near-IR ( $J H K$ and often $L$ ) images which are typically seeing-limited to $\sim 0.8$ resolution. Sources in the composite data set were analyzed for multiplicity over a separation range from the resolution limit (typically 100 - 300 AU depending on cloud distance and seeing) to a maximum separation of 2000 AU. These results are shown in Table 1.

These results show that binaries (and multiples in general) are common among embedded protostars, even given the relatively large minimum separation 
Table 1. Haisch et al. (2002, 2003) Protostellar Multiplicity Analysis

\begin{tabular}{lrrrc}
\hline Region & Min. Sep. (AU) & \# Observed & \# Binary/Multiple & Fraction (\%) \\
\hline Per & 320 & 9 & 1 & $11 \pm 11$ \\
Tau-Aur & 140 & 11 & 1 & $9 \pm 9$ \\
Cha I \& II & 100 & 17 & 3 & $18 \pm 10$ \\
Ser & 310 & 16 & 5 & $31 \pm 14$ \\
$\rho$ Oph & 125 & 38 & 10 & $26 \pm 8$ \\
& & & & \\
TOTAL & & & 20 & $22 \pm 5$ \\
\hline \hline
\end{tabular}

distances to which this survey was sensitive. The data also hint that protostars in different regions may have different multiplicity characteristics, but observations of more objects are needed to investigate this possibility with statistical significance. Haisch et al. (2003) determined that the multiplicity fraction of the embedded protostars was statistically indistinguishable from that of PMS stars observed in the Tau-Aur, $\rho \mathrm{Oph}$, and Cha regions once separation ranges and dynamic ranges were properly restricted. Furthermore, Haisch et al. (2003) also found that the multiplicity fraction of their protostellar sample is statistically indistinguishable from that of the Reipurth (2000) HH driver sample when similar separation limits are imposed on both groups. Many of the Reipurth objects have relatively close separations (sub-arcsecond) which were not probed by the Haisch et al. (2002; 2003) surveys. This and the relatively modest number of objects in the Reipurth survey result in it having a multiplicity fraction of $29 \%$ $\pm 14 \%$ when restricted to the separation range of the embedded protostellar sample (Haisch et al. 2003).

The Reipurth (2000) notion of disintegrating multiple systems may well apply in star forming regions even though there is no obvious dynamical evolution between the embedded protostellar, HH driver, and PMS stages. In this scenario, triples and higher multiples decay in only $\sim 10^{4} \mathrm{yr}$ after formation, so all of the observed protostars, HH drivers, and PMS stars will have already gone through this process. Furthermore, ejected stars travel at $1-3 \mathrm{~km} \mathrm{~s}^{-1}$ (Sterzik \& Durisen 2003; Delgado-Donate et al. 2003). This means that they travel at least $2000 \mathrm{AU}$ in $10^{4} \mathrm{yr}$, so they would also be beyond the maximum separation limits of all of the multiplicity surveys. However, it may be possible to witness the the disintegration of extremely young multiple systems by observing the youngest Class 0 protostars (at sub-mm or radio wavelengths).

Haisch et al. (2002) presented $1-10 \mu \mathrm{m}$ spectral energy distributions (SEDs) for both components of 2 binary systems, WL 1 in $\rho$ Oph and SVS 20 in Ser. The SEDs of the primary and secondary components were very similar to each other in both systems, suggesting that the primaries and secondaries have similar ages. This is consistent with the general result that components of PMS multiple systems have similar or identical ages (e.g., White \& Ghez 2001). Analyzing binary protostars increases the resolution of coevality measurements since these objects have lifetimes of only $\sim 10^{5} \mathrm{yr}$, at least an order of magnitude less than PMS stars. However, near-to-mid IR SEDs can be difficult to obtain 


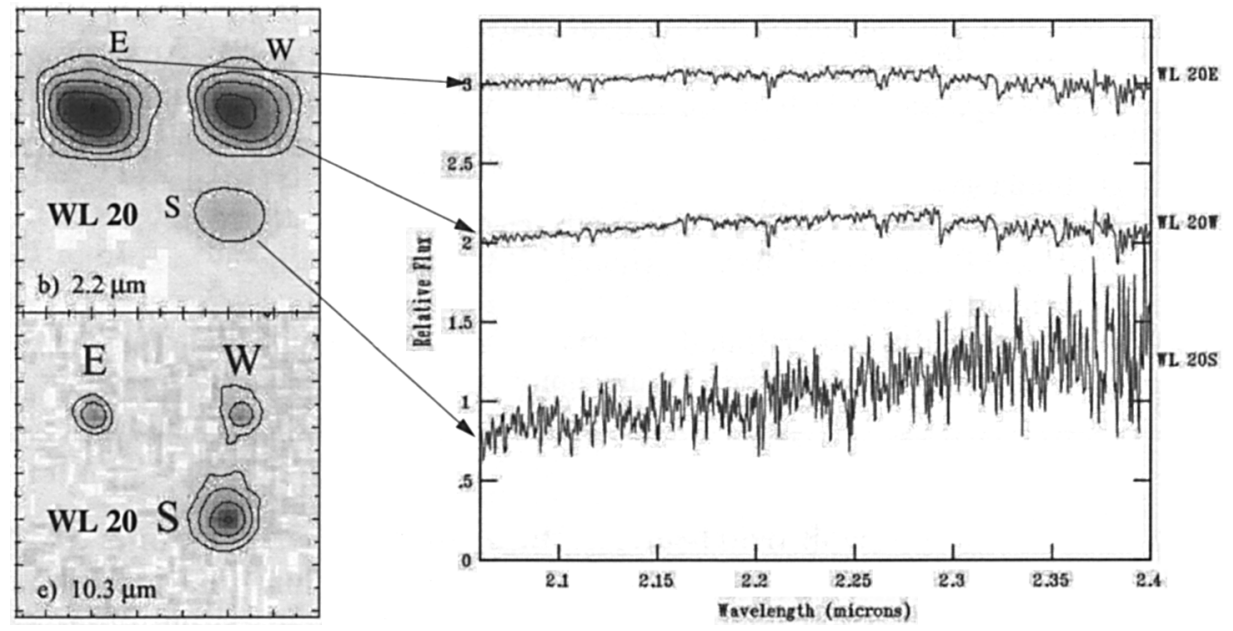

Figure 1. Near- and mid-IR images (left; Ressler \& Barsony 2001) and spectra (right; Barsony et al. 2002) of the WL 20 system.

and provide only a gross measure of age, so they are not the ideal tool for measuring the coevality of protostellar components with high precision.

\section{Lessons from Spectroscopy}

Near-IR spectroscopic observations of protostars have become increasingly common in recent years, and several recent studies provide unique insight to the properties of protostellar binary and multiple systems. In general, the photometric and SED properties of protostars and PMS stars are correlated with their $2 \mu \mathrm{m}$ spectra (e.g., Greene \& Lada 1996). This can be seen by comparing $K$ and $N$ band images of the components of the WL 20 system in $\rho$ Oph (from Ressler \& Barsony 2001) to their $2 \mu \mathrm{m}$ spectra (from Barsony, Greene, \& Blake 2002), as shown in Figure 1.

It is worth noting that Ressler \& Barsony (2001) found the young triple system WL 20 to have SEDs consistent with those of 2 PMS stars (WL $20 \mathrm{E}$ and $\mathrm{W}$ ) and one embedded protostar (WL $20 \mathrm{~S}$ ). The $2 \mu \mathrm{m}$ spectra of WL $20 \mathrm{E}$ and $\mathrm{W}$ both show absorption features indicative of late-type PMS stars, while that of WL $20 \mathrm{~S}$ shows only a heavily reddened featureless continuum (see Fig. 1). These characteristics are typical for moderate resolution and modest signal-to-noise spectra of PMS stars and protostars, respectively. The spectral lines of protostars are usually strongly veiled by strong continuum emission from circumstellar matter. This emission is also responsible for the mid-IR excesses of these objects, and both reddening and its intrinsic low temperature cause it to increase steeply from near- to mid-IR wavelengths.

WL 20 is not the only young multiple system to harbour both PMS and protostellar components. Prato, Greene, \& Simon (2003) obtained near-IR spectra of both components of 17 binary systems located in four nearby star forming regions. This sample was selected to be mostly PMS stars, and most systems 
are composed of components which both show PMS spectra. However, 3 of the 17 systems (AS 205, DoAr 24E, and AS 353) have 1 component with a PMS spectrum and 1 with a protostellar spectrum. These mixed spectra suggest that these systems (along with WL 20 also) may not be coeval, but there may be another explanation for these differences. It is possible that both components may be PMS stars, and a recent event (such as a dynamical encounter) may have triggered substantial accretion in only one of the systems' members.

\section{Thoughts for the Future}

The basic multiplicity properties of protostellar systems are just now being observed and analyzed, and there is much more fruitful work which could be done relatively easily at this time. It would be very worthwhile to survey more of these Class I (and flat spectrum) protostars in nearby clouds in order to ascertain better whether they truly have the same binary / multiplicity frequencies as PMS stars, especially those residing in the same clouds as the protostars. New searches should also probe smaller minimum separation distances in order to be more comparable to the existing high resolution studies of PMS stars. Near-IR speckle and adaptive optics (AO) observations may be very effective for achieving these goals, but they may also be hampered somewhat by the fact that many embedded protostars are spatially extended (but usually centrally peaked) at $2 \mu \mathrm{m}$ due to their large amounts of scattered light (e.g., see Tamura et al. 1991; Allen et al. 2002).

I heartily thank the many people who contributed to my understanding of this subject,and I also thank the organizers of IAU Symposium 221 for planning and conducting an outstanding meeting. These include Mary Barsony, Tyler Bourke, Michael Burton, Karl Haisch, Ray Jayawardhana, Lisa Prato, Michal Simon, Steve Stahler, Russel White, and many others.

\section{References}

Allen, L. E., Myers, P. C., Di Francesco, J., Mathieu, R., Chen, H., \& Young, E. 2002, ApJ, 566, 993

Barsony, M., Greene, T. P., \& Blake, G. A. 2002, ApJ, 572, L75

Delgado-Donate, E. J.; Clarke, C. J.; Bate, M. R. 2003, MNRAS, 342, 926

Duchêne, G. 1999, A\&A, 341, 547

Ghez, A. M., McCarthy, D. W., Patience, J. L., \& Beck, T. L. 1997, ApJ, 481, 378

Greene, T. P. \& Lada, C. J. 1996, AJ, 112, 2184

Haisch, K. E., Barsony, M., Greene, T. P., \& Ressler, M. E. 2002, AJ, 124, 2841

Haisch, K. E., Greene, T. P., Barsony \& Stahler, S. W. 2003, AJ, accepted

Melo, C. H. F. 2003, A\&A, in press

Petr, M. G., Coude Du Foresto, V., Beckwith, S. V. W., Richichi, A., \& McCaughrean, M. J. 1998, ApJ, 500, 825 
Prato, L., Greene, T. P., \& Simon, M. 2003, ApJ, 584, 853

Reipurth, B. 2000, ApJ, 120, 3177

Reipurth, B., Chini, R., Krugel, E., Kreysa, E., \& Sievers, A. 1993, A\&A, 273, 221

Ressler, M. E. \& Barsony, M. 2001, AJ, 121, 1098

Sterzik, M. F. \& Durisen, R. H. 2003, A\&A, 400, 103

Tamura, M., Gatley, I., Waller, W., \& Werner, M. W. 1991, ApJ, 374, L25

White, R. J. \& Ghez, A. M. 2001, ApJ, 556, 265 Symposium, 200, 464 